\author{
Military Technical College \\ Kobry El-Kobbah, \\ Cairo, Egypt
}

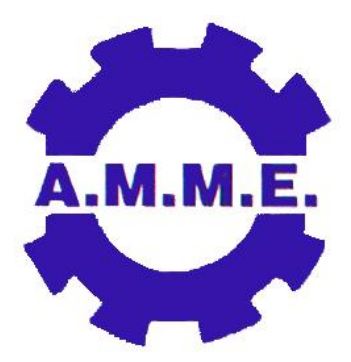

14th International Conference on

Applied Mechanics and

Mechanical Engineering.

\title{
Evaluation of wind noise emitted from vehicle windows
}

\author{
By
}

\author{
Essam M. M. Allam *
}

\begin{abstract}
:
The characteristics of the wind noise radiated from vehicle windows of a passenger car depend on its shape, cruising speed, wind direction towards the vehicle and the natural wind condition. For these factors, the vehicle shape is the most important controllable factor. The exterior shape, which significantly affects the wind noise, is decided in the early design stage of the actual vehicle development process. A vehicle driver may be particularly annoyed at open-windows conditions. Thus, a measurement campaign has been carried out in order to characterize the aerodynamic noise generated by air flows through vehicle windows. Noise levels have been measured at the driver ears by varying vehicle speed and driver-side window opened area. Experimental results have shown that the noise is mainly depending on the following physical parameters: vehicle speed, car internal volume, and window open area that creates large pressure differences between the inside and outside of the car. The Light hill acoustic analogy is employed to estimate the far field wind noise
\end{abstract}

\section{Keywords:}

Aerodynamic noise, Wind noise, Noise source 
* Automotive and Tractors Engineering, Faculty of Engineering, Helwan University, Cairo, Egypt

\section{Introduction:}

Noise refers to the unwanted sounds and vibrations and the noise generated by moving vehicles has many different sources. For example, noise of conventional passenger cars can be emitted from various places such as aerodynamic friction, engine vibrations, tire/road contact, engine acceleration, braking, horns and vehicle theft alarms, exhaust pipe, transmission box, and vehicle window opened area. Therefore, automobile aerodynamic and wind noise have received more attention in recent years [1] as a result of design improvements that reduce the sources of noise significantly and to increase operating speed for highway driving [2, 3]. Also, the automobile manufacturers have increased their efforts to design and build quieter cars, and reduce vehicle interior noise through addition of extra door seals and use of acoustic insulation package. Low noise emission engines and sound insulation cowlings make contribute to vehicle improvement.

The lowest noise level is produced from the rolling tyre and is contrasted by means of sound absorbing pavements and an appropriate design of tyre shape [1]. Exhaust noise, on the other hand, may be reduced by means of multi-stage passive mufflers and used catalytic. In addition, aerodynamic noise, which is particularly remarkable when vehicle windows are opened, may be reduced by peculiar vehicle shape design [3]. To counteract that, noise sources have to be reduced, noise transfer paths have to be damped even further, leaks have to be avoided, and sound insulation and absorption packages have to be more effective. The new environmental restrictions have positive impact on the fuel consumption, carbon dioxide emissions and vehicle interior noise.

The side windows of an automobile are especially important for the interior noise level and represent one of the major radiators of wind noise into the passenger compartment. In this paper, a measurement campaign has been led to evaluate the aerodynamic noise characteristics at different open-windows conditions and different vehicle velocities. Measurements are carried out in the region near the driver ears as it represents the most important place.

\section{Aerodynamic and wind Noise}

Aerodynamic noise is the noise caused by temporal fluctuations of airflow around the body of a moving automobile. On the other hand, wind noise, which varies from vehicle to vehicle, is created by the air flowing over the car and hitting protrusions such as rearview mirrors, roof racks, and driving with open windows. Wind noise is also controlled by the amount of sound insulation installed by the manufacturer. Meanwhile, the created noise, at certain speed, when driving with one side open window is different from that of the two side open window. When the vehicle travels at high speeds, fluctuations in the separation vortexes around the side-view mirror results in mirror surface vibration while the rear visibility is adversely affected.

\section{A measurement method}

Noise levels at different open windows conditions have been measured using a polo car as shown in Figure 1. the used measurement microphone is IEC 61672: 2003 and this mandates the inclusion of an A-frequency-weighting filter and also describes other 
frequency weightings of ' $C$ ' and ' $Z$ ' (zero) frequency weightings. The older ' $B$ ' and ' $D$ ' frequency-weightings are now obsolete and are no longer described in the standard. The microphone is adjusted according to the following three conditions. First, position (a) is for slightly-downward street and gears set on neutral position. Second, position (b) is for insulating cowlings. Finally, position (c) is for silent tyres. Measurement microphone has been equipped with a windscreen protection and has been positioned near the windowside driver ear (see Figure 1). Tests have been carried out for different driver-side window open area and vehicle velocity conditions

\section{Experimental Results}

Aerodynamic noise levels relative to different vehicle speed and window opening conditions are reported in Table 1. It can be seen that the aerodynamic noise values increase with the vehicle speed and window opened area. The influence of vehicle speed on the total noise of the window beside driver is shown in Figures 2 and 3. However the noise level increases with the increase of the vehicle speed, it also increases with the open area of the window, at certain speed. Figure 4 shows the variation of the noise level with the vehicle speed for window open area of $25 \%$ and $100 \%$. The maximum difference between the two conditions occurs at vehicle speed of $90 \mathrm{~km} / \mathrm{hr}$. Figure 5 illustrates the aerodynamic noise at complete closed and complete open window conditions. It can be seen that the maximum difference of the noise level lies between 8 to $12 \mathrm{db}$.

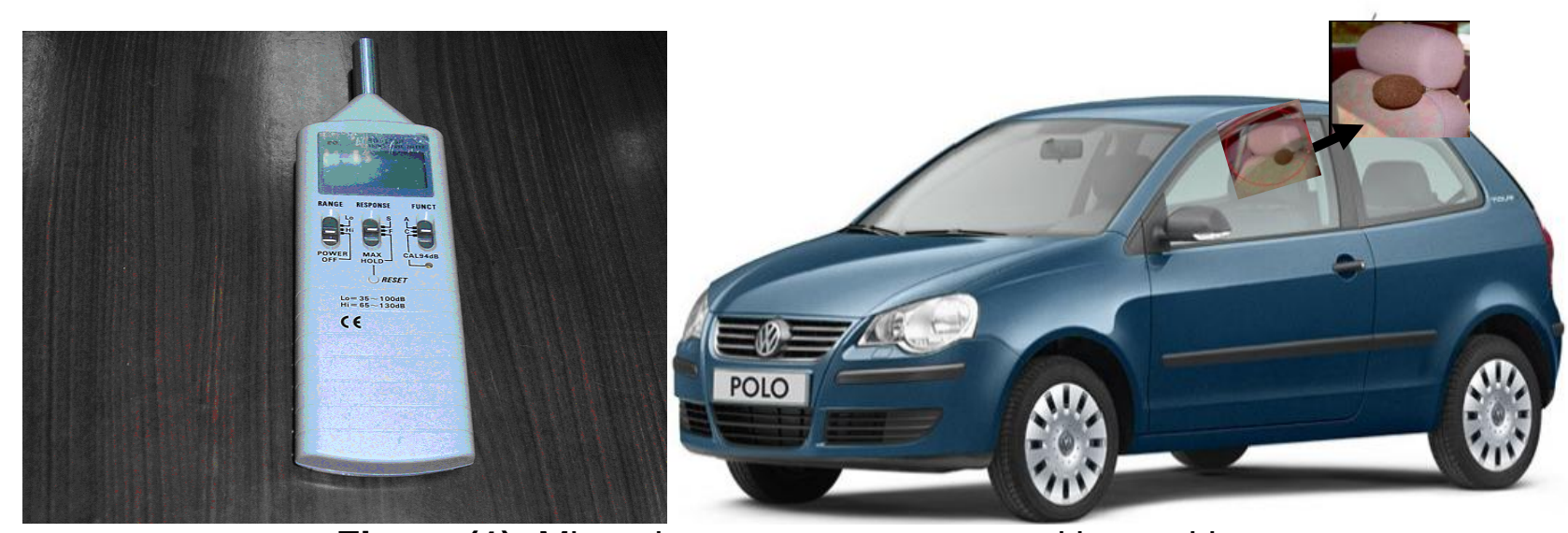

Figure (1): Microphone measurement and its position 


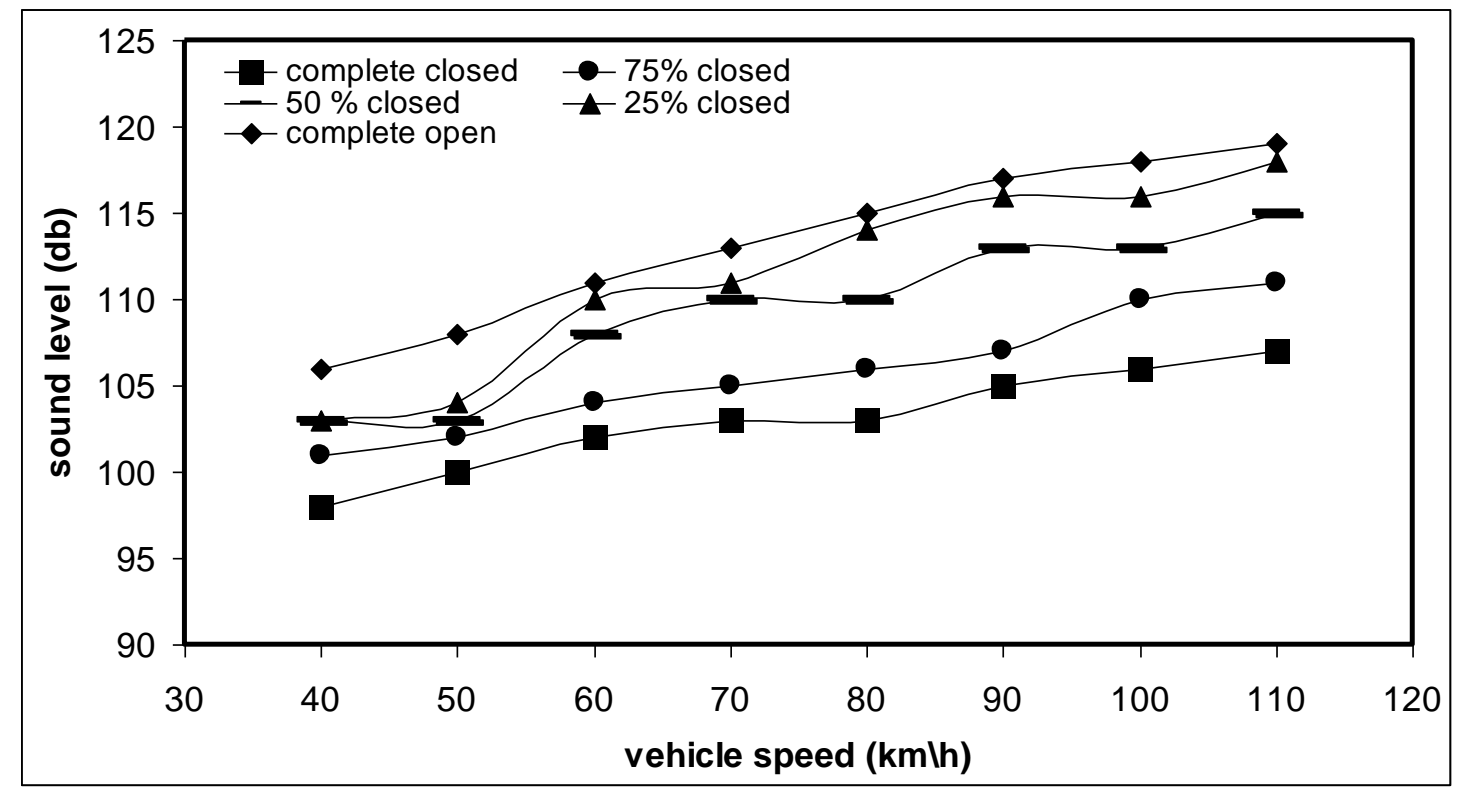

Figure (2): Measured aerodynamic noise spectra (window opened area and vehicle speed conditions)

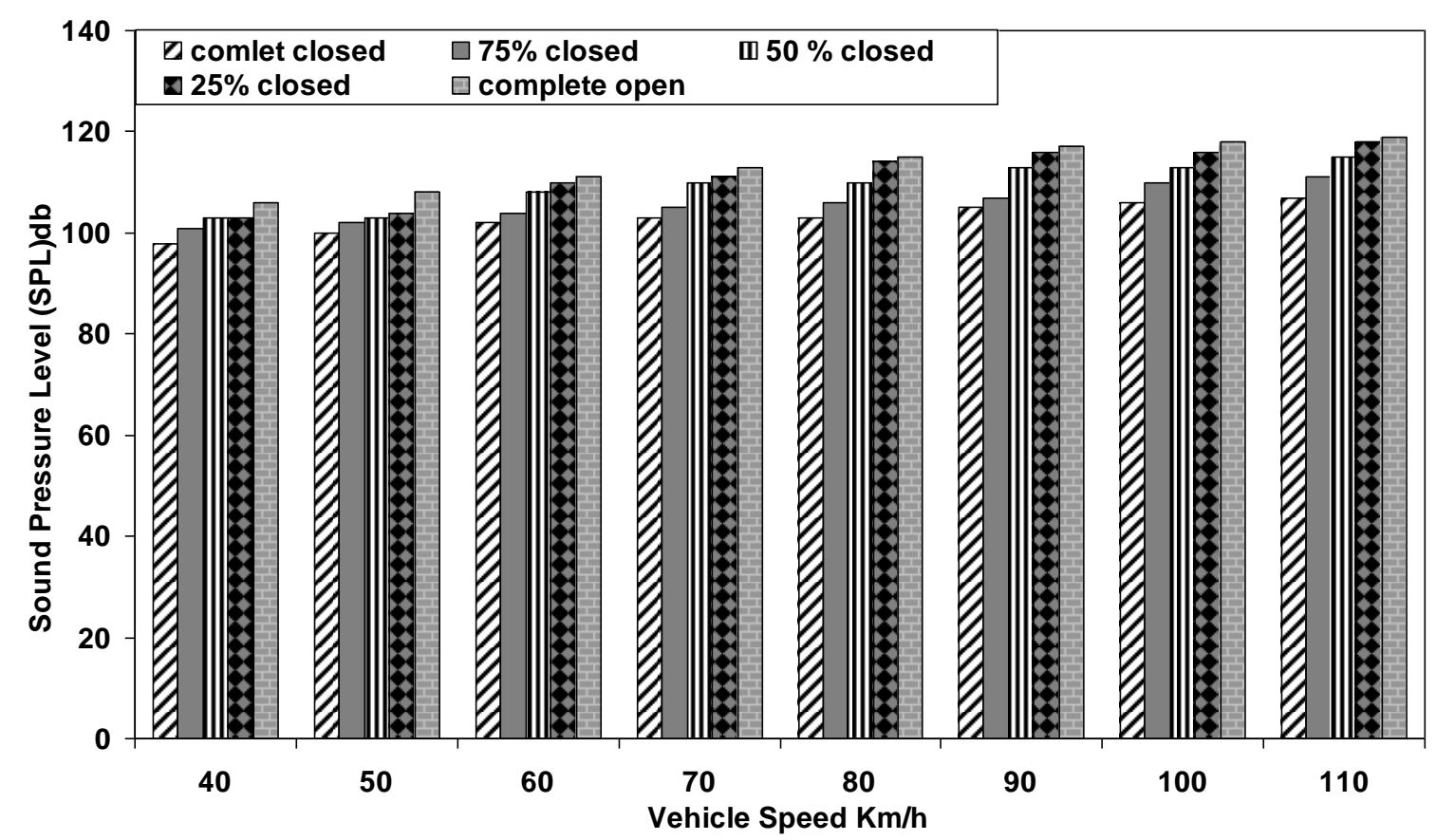

Figure (3): Measured aerodynamic noise spectra relative to car (window opened area and vehicle speed extreme conditions 


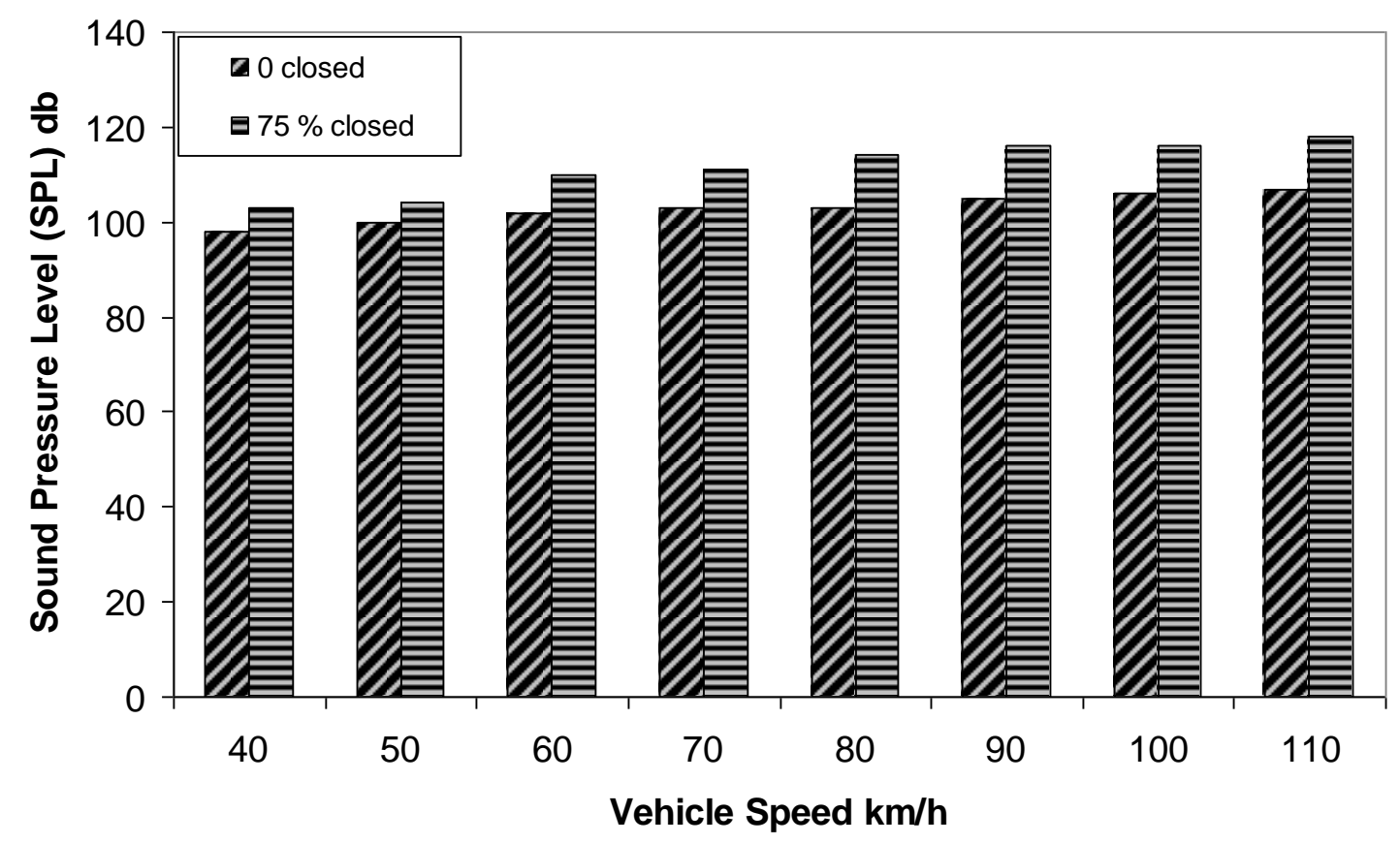

Figure (4): Measured aerodynamic noise spectra relative to car (window $25 \%$ and $100 \%$ opened area and vehicle speed conditions

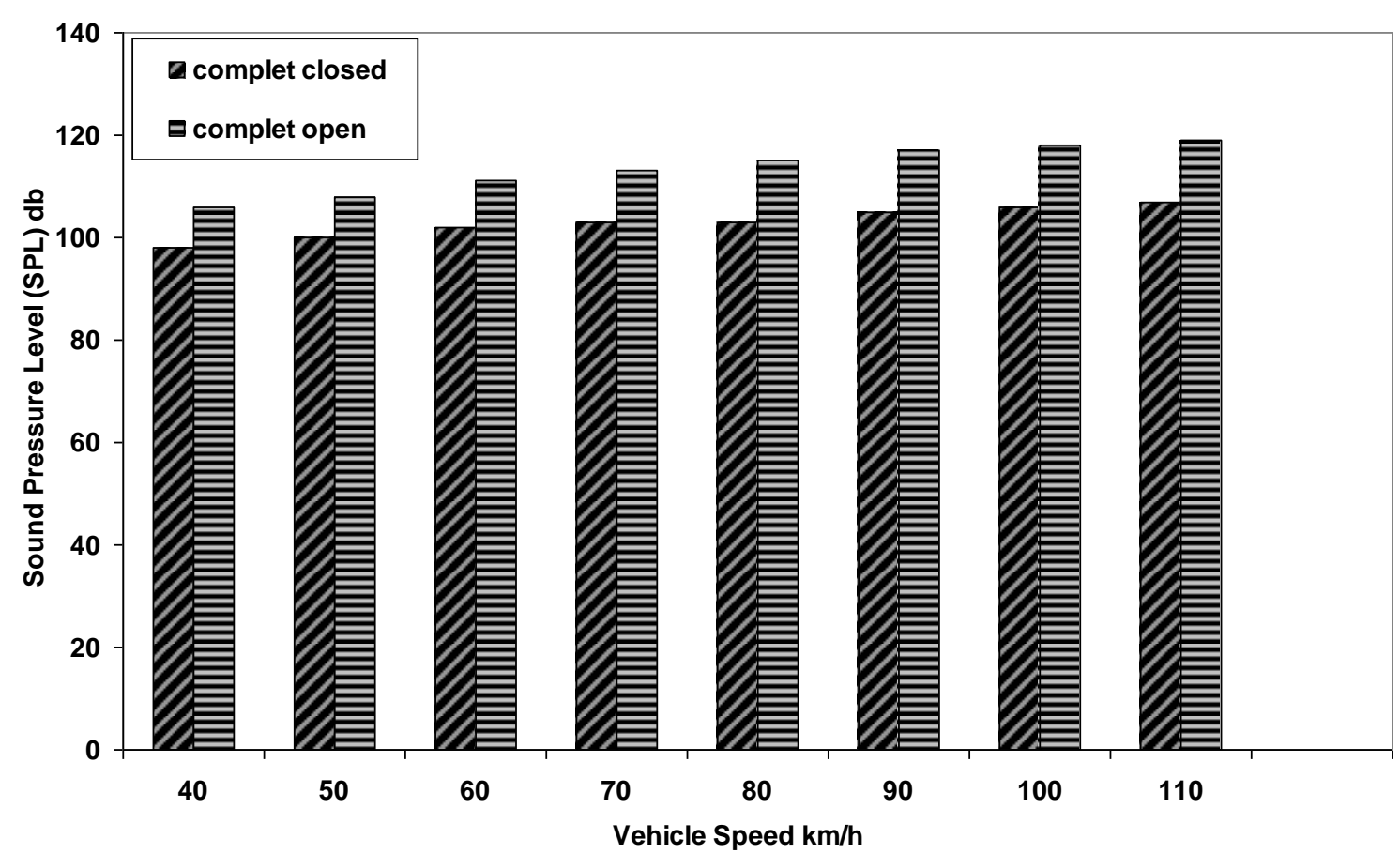

Figure (5): Measured aerodynamic noise spectra relative to car (window $100 \%$ closed and $100 \%$ opened area and vehicle speed conditions

Table (1) : Measured and estimated aerodynamic noise

\begin{tabular}{|c|l|l|l|l|l|l|l|l|}
\hline vehicle speed / noise $(\mathrm{db})$ & 40 & 50 & 60 & 70 & 80 & 90 & 100 & 110 \\
\hline complete closed & 98 & 100 & 102 & 103 & 103 & 105 & 106 & 107 \\
\hline $75 \%$ closed & 101 & 102 & 104 & 105 & 106 & 107 & 110 & 111 \\
\hline $50 \%$ closed & 103 & 103 & 108 & 110 & 110 & 113 & 113 & 115 \\
\hline $25 \%$ closed & 103 & 104 & 110 & 111 & 114 & 116 & 116 & 118 \\
\hline
\end{tabular}




$111=113$

115

117

$118=119$

\section{Conclusion:}

Several opened-area of an automobile window are tested for measuring noise. An experimental investigation on aerodynamic noise perceived by car driver in open-window conditions has been carried out. A measurements campaign has been led by varying vehicle speed and driver-side window open area. Automotive internal noise increases with the increase of the vehicle window opened area and car velocity. The effect of the opened portion of a car window plays an important role on the induced noise level.

\section{References:}

[1] F. Rossi, "Active Noise Control Technique to Improve Energy Efficiency", Proceedings of Energy and Environment, Capri, Volume 13, Issues 2-4, March, Pages 620-623, 2002.

[2] F. Rossia and A. Nicolinia Theoretical and Experimental investigation of the aerodynamic noise generated by air flows through car windows" a Dipartimento dilngegneria Industriale,Università degli Studi di Perugi Via G. Duranti 67, 06125 Perugia, Italy, 2003.

[3] S. Phillips, S. Kollamthodi and P. Nelson, "Investigations of Tyre/Road Noise Draft Report", Project Report PR/SE/272/2001 S032J/VB, Transport Research Laboratory,Crowthorne, U.K, 2001.

[4] AL Marsden, M Wang, Mohammadi, P "Shape Optimization for Aerodynamic Noise Control", in Center for Turbulence Research - Center for Turbulence Research, Annual Research Briefs, pp.241-247, 2003

[5] L.L. Beranek, I.L. Ver, "Noise and Vibration Control Engineering, John Wiley \& Sons, New York, 1992.7. S. Haruna et al., "Numerical study of aerodynamic noise radiated from a three-dimensional" wing, SAE Paper, 92034, 1992.

[6] S. Adachi et al., "Computation of aerodynamic sound radiation from Flow" past a wing, DGLR/AIAA14th Aeroacoustic Conference, DGLR/AIAA-92-02-157, 1992.

[7] M. Zhu et al., "A numerical study of wind noise around front pillar", SAE Paper, 930296, 1993.

[8] C. Kato et al., "Numerical simulation of aerodynamic sound radiated from low Mach number turbulent wakes", Computational Aeroacoustics, Fluid Engineering Division, vol. 219, ASME, 1995.

[9] P. Bergamini et al., "Computational prediction of vehicle aerodynamic noise byintegration of a CFD technique with Lighthill's acoustic analogy", SAE Paper, 970401, 1997.

[10] P.G. Buning et al.,"Numerical simulation of the integrated space shuttle vehicle in ascent", AIAA-88- 4359-CP, 1988 .

[11] K. Fujii, "Unified zonal method based on the fortified solution Algorithm" J. Comput. Phys. 118, pp. 92-108, 1995.

[12] M.M. Rai, " A conservative treatment of zonal boundaries for Euler equation calculation", AIAA 22nd Aerospace Sciences Meeting, AIAA-84-0164, Reno, 
Nevada, January, 1984.

[13] Z.J. Wang, [1995] "A fully conservative interface algorithm for overlapped grids". J. Comput. Phys. 122, pp. 96-106.

[14] T. Miyauchi, M. Tani, "Numerical analysis of homogeneous and isotropic turbulence by using the third order finite- difference method (study in wave numerical space)", JSAE 53.486, pp. 388-392, 1987

[15] T. Kawamura et al., " Computation of high Reynolds Number Flow around a circular cylinder with surface roughness. Fluid Dyn. Res. 1, pp. 145-162. , 1986.

[16] R. Himeno et al., Numerical " analysis of the airflow around automobiles using multi block structured grids", SAE Paper, 900319, 1990. 